\title{
Thermal and mechanical improvement of aluminum open-cells foams through electrodeposition of copper and graphene
}

\author{
Alessandro Simoncini*, Nadia Ucciardello, and Vincenzo Tagliaferri \\ University of Rome “Tor Vergata”, Dipartimento di Ingegneria dell'Impresa "Mario Lucertini”, Via del Politecnico 1, \\ Rome 00133, Italy
}

Received 4 October 2016 / Accepted 10 November 2016

\begin{abstract}
Thanks to its planar structure, graphene is characterized by unique properties, such as excellent chemical inactivity, high electrical and thermal conductivity, high optical transparency, extraordinary flexibility and high mechanical resistance, which make it suitable in a very wide range of applications. This paper details the state of the art in graphene coating applied to aluminum open-cells foams for the improvement of their mechanical and thermal behavior. Metallic foams are highly porous materials with extremely high convective heat transfer coefficients, thanks to their complex structure of three-dimensional open-cells. Graphene nanoplatelets have been used to improve thermal conductivity of aluminum foams, to make them better suitable during heat transfer in transient state. Also, an improvement of mechanical resistance has been observed. Before electrodeposition, all the samples have been subjected to sandblasting process, to eliminate the oxide layer on the surface, enabling a better adhesion of the coating. Different nanoparticles of graphene have been used. The experimental findings revealed a higher thermal conductivity for aluminum open cells foams electroplated with graphene. Considered the relatively low process costs and the improvements obtainable, these materials are very promising in many technological fields. The topics covered include surface modification, electrochemical plating, thermo-graphic analysis.
\end{abstract}

Key words: Graphene electrodeposition, Open-cells aluminum foams, Thermal characterization

\section{Introduction}

Heat removal has become a crucial issue for continuing progress in many technological fields owing to increased levels of dissipated power. The search for materials that conduct heat well has become essential for the design of the next generation of heat exchangers. Due to their advantageous characteristics, such as low production costs, wide potential window, high thermal conductivity and mechanical strength, inert electrochemistry [1], carbon materials have been widely used in many technological fields [2, 3]. The advantages of carbon materials combined with those of nanostructured materials, such as carbon nanotubes $[4,5]$, carbon nano-fibers [6], highly ordered mesoporous carbons [7, 8], etc., have drawn much more attention because of their unusual electronic properties and ability to improve catalytic properties [9]. In solid materials heat is carried by acoustic phonons, which are ion-core vibrations in a crystal lattice, and electrons so that the thermal conductivity coefficient $(\lambda)$ is the sum of the phonon $\left(\lambda_{\mathrm{p}}\right)$ and electron $\left(\lambda_{\mathrm{e}}\right)$ contributions. While in metals $\lambda_{\mathrm{e}}$ is dominant, heat conduction

*e-mail: alessandro.simoncini@uniromaz.it in carbon materials is usually dominated by phonons, even for graphite [10], which has metal-like properties [11]. The phonon thermal conductivity is expressed as $\lambda_{\mathrm{p}}=\Sigma_{j} \int C_{j}(\omega) v_{j}^{2}(\omega) \tau_{j}(\omega) \mathrm{d} \omega$, where $j$ is the phonon polarization branch, that is two transverse acoustic branches and one longitudinal acoustic branch; $v$ is the phonon group velocity, which, in many solids, can be approximated by the sound velocity; $\tau$ is the phonon relaxation time, $\omega$ is the phonon frequency and $C$ is the heat capacity. The thermal conductivity values obtainable for carbon materials span a very wide range from $\sim 0.01 \mathrm{~W} / \mathrm{mK}$ to $10,000 \mathrm{~W} / \mathrm{mK}$ in type-II diamond at a temperature of approximately $77 \mathrm{~K}$ [12]. The exfoliation of graphene and the availability of high quality few-layer graphene (FLG) led to experimental observations of the evolution of thermal properties as the system dimensionality changes from 2D to 3D. First measurements of the thermal properties of graphene [13-16] revealed a thermal conductivity above the bulk graphite limit - high quality pyrolytic graphite has an in-plane thermal conductivity $\left(\lambda_{/ /}\right)$of $\sim 2000 \mathrm{~W} / \mathrm{mK}$ at room temperature, while its cross-plane thermal conductivity $\left(\lambda_{\perp}\right)$ is more than two orders of magnitude smaller - which awakened strong interest in the thermal properties of this 

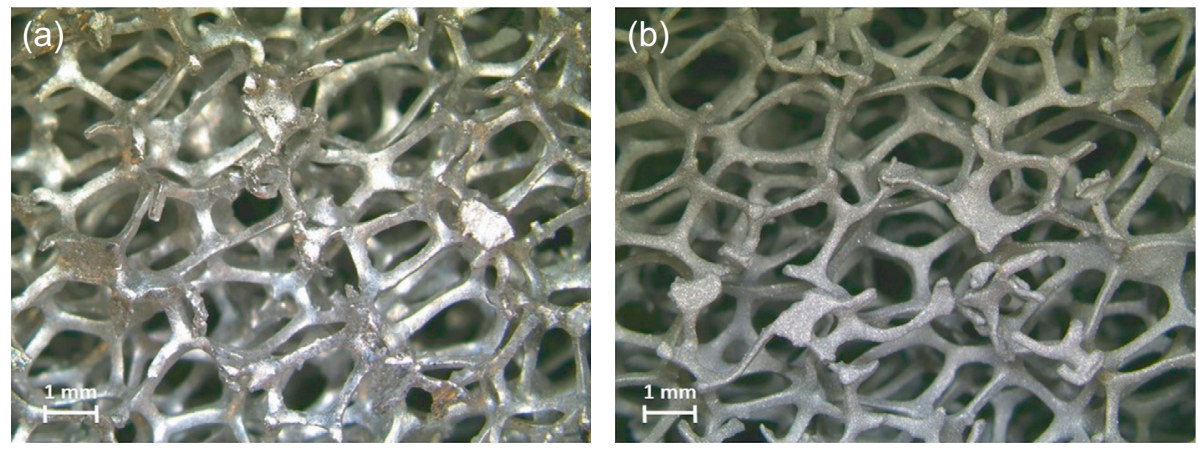

Figure 1. Aluminum foam surface: (a) before sandblasting; (b) after sandblasting.

material and in crystals of lower dimensionality. The recent discovery of graphene [17] has opened a new era of twodimensional fundamental science and potential technology $[18,19]$. As the basic structure of all graphitic forms, graphene is a building block for carbon materials of all other dimensionalities, such as 0D fullerene, 1D nanotube, and 3D graphite. Because of its properties such as large specific surface area [20], high thermal and electrical conductivities [21], great mechanical strength [22], graphene has attracted a growing interest, founding potential applications in many fields such as nanocomposites [23], batteries [24], supercapacitors [25], nanoelectronics [26] and sensors [27], etc. These peculiar properties make graphene an excellent additive to dramatically enhance the mechanical, electrical and thermal properties of compounds [28]. Through these years, considerable efforts were made to create deposits of a graphene monolayer with the purpose of giving exceptional properties to the substrate on which it was placed $[29,30]$. Several deposition methods have been proposed to fabricate two-dimensional graphene sheets on conductive substrates, such as chemical vapor deposition and thermal decomposition [31,32]. However, most of these are burdensome for the assembly of graphene layers because they employ high temperatures and complex instrumentation [33]. The electrodeposition process of graphene on a metal substrate allows exploiting the superior properties of this nanomaterial to improve those of the substrate. The present paper describes the developments in the deposition of graphene nano-platelets on aluminum open cells foam by electrochemical method. Aluminum open cells foam have been chosen as substrate mainly for their excellent properties such as lightweight, high impact resistance and extremely high convective heat transfer coefficient. Due to their size (small section, high length) the branches of the foam are not particularly efficient for heat exchange through conduction. For this reason, the use of a surface graphene layer allows increasing the efficiency of the foam, namely reaching higher temperature in lesser time. A 6101-T6 aluminum alloy was chosen for the samples. In this work, the novel method of electro-deposition at low temperature [3] has been applied to 10 PPI (pore per inch) aluminum alloy foam to improve their mechanical strength and thermal behavior. The extremely porous structure of open cells metal foam, with actual density greater than $90 \%$, let them reach particularly high convective heat transfer coefficient, making them promising as compact air-based heat exchangers [34]. The paper also focuses on the nano-platelets disposition mechanism, with special emphasis on the improvement of thermal conduction and on the functional uses of these materials for heat dissipation. In the end, different nano-particles have been used for the combined electrodeposition process. Infrared (IR) thermo-graphic analyses have been led to compare the enhancements in thermal conduction of the foam.

\section{Materials and experimental procedure}

All the aluminum samples were obtained from the same block, produced by ERG Aerospace Corp. (2015), and manufactured with the same parallelepiped shape $(30.5 \pm 0.7) \times(50.6 \pm 0.5) \times(0.8 \pm 0.1) \mathrm{mm}$. The surface preparation was carried out using a sandblasting machine, which exploits tiny corundum spheres, at high pressure, on the sample surface. The surface preparation has been led using grain $80(180-212 \mu \mathrm{m})$ as particle size of the sand, 6 bars for the pressure and $8 \mathrm{~s}$ as duration of execution of the process. This treatment enables to easily remove the oxide layer that is formed on the aluminum surface, which is the cause of an imperfect and irregular coating, varying the surface roughness profile that affects the electrodeposition process (Figure 1).

Since the rate at which the oxide layer is regenerated is particularly high, the electrodeposition has been realized immediately after the sandblasting process. Due to the extreme volatility of graphene, the electrodeposition of copper only before and after the combined electrodeposition process of copper and graphene became necessary. Given the high electron affinity between the two materials, the first copper layer has the function to entirely cover the substrate, making the graphene deposition process easier, while the last layer prevents it to go outside. So, each sample was electrodeposited in three steps, or phases: first copper, then graphene and copper and, in the end, copper again (Figure 2 [3]).

For the process of copper plating, a copper sulfate based solution was used as acidic electrolytic bath. The sacrificial anode used for the entire process was composed of two copper plates $(75 \times 76 \times 15 \mathrm{~mm})$ connected to each other to completely cover the sample, allowing the best possible results. The anode was thus also designed to make easily accessible that part of the aluminum sample, which is always above the 
(a)

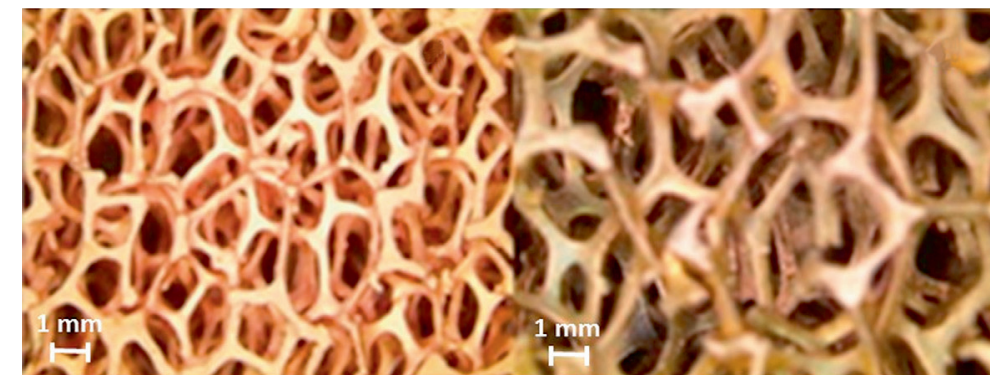

(c)

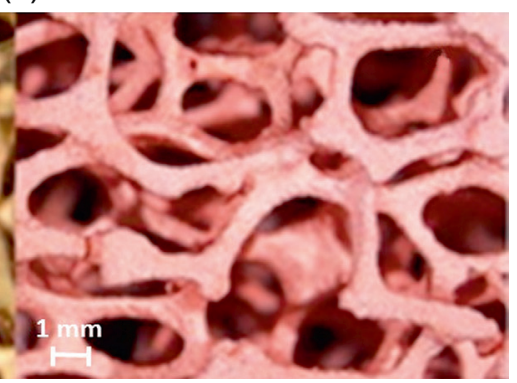

Figure 2. Electrodeposition phases: (a) copper; (b) copper and graphene; (c) copper.

free surface of the solution, because connected to the terminal of the current generator. Two different kinds of solution were exploited in the three different phases of electrodeposition. Regarding the deposition of copper only (first and third phase) the electrolytic bath used was an acidic bath consisted of: $1.25 \mathrm{M} \mathrm{CuSO}_{4}, 0.61 \mathrm{M} \mathrm{H}_{2} \mathrm{SO}_{4}$ and $\mathrm{Cl}^{-} 50 \mathrm{ppm}$. The second phase instead was characterized by a basic solution of: $1.25 \mathrm{M} \mathrm{CuSO} 4, \mathrm{Cl}^{-} 50 \mathrm{ppm}$ and GNP (graphene nano-platelets), provided by NANESA s.r.l. (Italy). GNP consists of small stacks of graphene obtained by exfoliation of expanded graphite, with a carbon content of $98 \%$ and less than $1 \%$ of acidic residual. Two different GNP have been used:

- G2N, with an average flake thickness of $10 \mathrm{~nm}$ ( $\sim 30$ layers), an average particle size of $5 \div 50 \mu \mathrm{m}$ and specific surface area $>30 \mathrm{~m}^{2} / \mathrm{g}$.

- G3N, with an average flake thickness of $9 \mathrm{~nm}$ ( 25 layers), an average particle size of $15 \mu \mathrm{m}$ and specific surface area $>40 \mathrm{~m}^{2} / \mathrm{g}$.

To maximize the amount of graphene deposited during the second phase, the following process conditions have been used:

- electrolytic bath temperature of $60{ }^{\circ} \mathrm{C}$;

- electrolytic current of $0.05 \mathrm{~A}$;

- $0.336 \mathrm{~g}$ of GNP dispersed in solution.

The amount of GNP dispersed in solution and galvanic parameters used are the results of previous optimization works $[2,3]$. The baths were kept in agitation with a magnetic agitator, located inside the electrolytic cell. The agitation was set at $3 \mathrm{rpm}$. After electrodeposition, all the samples were put under vacuum. In this way, the integrity of the surface of the coating was prevented by possible alterations caused by the interaction of the copper with the atmosphere. In fact, the presence of these alterations, for example the oxidation of the copper surface or the formation of any other possible thermal barriers, could have modified the results of the thermal tests. Compression tests were leaded on each sample in a MTS Alliance RT50 testing machine with a $10 \mathrm{kN}$ load cell, setting a constant deformation rate of $5 \mathrm{~mm} / \mathrm{min}$. Conductivity tests were leaded with a IR thermal imaging camera, in order to evaluate which nanoparticle better improves the thermal conductivity of the sample, once electrodeposited on the surface. Convective thermal measurements, through forced air convection, were led to evaluate the improvements given by the GNP electrodeposition on the metal foams samples.

\subsection{GNP/substrate interface model}

The connection of graphene with other materials is a subject of both fundamental science and practical interest. Knowledge of interface coupling mechanics can help in understanding graphene thermal link to matrix materials. For what concerns the evaluation of the amount of graphene deposited during the 2 nd phase and the disposition of the platelets with respects to substrate, the diffusivity of each sample was determined by flash method [35]. The analysis of the thermal diffusivity allowed to determine how the GNP planes are arranged during the electrodeposition and which set-up enables to maximize graphene amount during the second phase. This study was necessary because a graphene multilayer (like our GNP) presents thermal properties depending on whether the incident heat flux is normal or parallel to the surface $[13,15]$. In a multilayer graphene lattice, heat is carried mostly by longitudinal acoustic (LA) and transverse acoustic (TA) phonons, and contributions of out-of-plane acoustic (ZA) phonons are negligible due to their small group velocity $(v \rightarrow 0)$ in the Brillouin zone center and large $\gamma[36,37]$. Considering the case in which: the heat flux is parallel to the surface, graphene thermal conductivity would be between 2000 and $5000 \mathrm{~W} / \mathrm{mK}$ [38]; obviously, the graphene thermal conductivity decreases with increasing thickness, approaching the bulk graphite limit. The comprehension about how the GNP planes are arranged on the substrate, during the combined deposition process, is fundamental for every possible future applications of the technology developed, according to the need of insulation or conduction that one wants to pursue. To verify the arrangement of the thin layer of graphene, an electro-thermal analogy has been applied, considering the different coatings disposed in series with the aluminum substrate. As shown in Figure 3, the 1st and 3rd phases are represented as a simple resistor placed in series with the one of the aluminum substrate, while the second phase has been shown schematically as two resistors placed in parallel. This choice is due to the fact that the electrodeposition of copper and graphene occurs simultaneously. An experimental campaign on 6000 -series aluminum plates was made specifically for the 


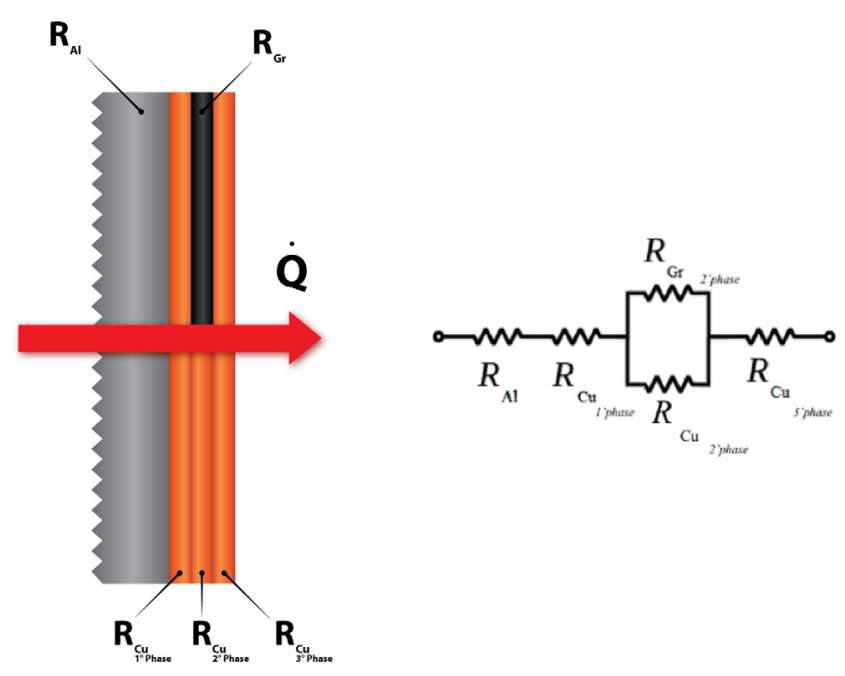

Figure 3. Electro-thermal scheme for the different phases coatings.

evaluation of the arrangement of GNP planes on the substrate. All the aluminum plates were prepared in the same way and, for what concerns the evaluation of the graphene amount and disposition during the 2 nd phase, the diffusivity of each sample was determined through flash method.

As regards the interface of graphene with the copper substrate, since the thermal boundary resistance $\left(R_{\mathrm{B}}\right)$ of graphene with many materials (mainly copper) is extremely low, the contact resistance has been neglected in the electrothermal analogy, even if $R_{\mathrm{B}}$ has a non-zero value considering perfect interfaces [39]. This approximation is valid for both the nanoparticles studied $(\mathrm{G} 2 \mathrm{~N}, \mathrm{G} 3 \mathrm{~N})$, because neither the cross-plane conductance nor $R_{\mathrm{B}}$ reveals a strong dependence on the thickness of FLG or the nature of the metal substrate $[40,41]$. Therefore, the thermal resistance of the coating can be expressed by the following equation:

$$
R_{\mathrm{tot}}=\sum R=R_{\mathrm{Al}}+R_{\mathrm{Cu}}^{1^{\circ} \text { phase }}+R_{\mathrm{Cu}+\mathrm{Gr}}^{2^{\circ} \text { phase }}+R_{\mathrm{Cu}}^{3^{\circ} \text { phase }}
$$

where:

$$
\frac{1}{R_{\mathrm{Cu}+\mathrm{Gr}}^{2^{\circ} \text { hase }}}=\frac{1}{R_{\mathrm{Cu}}^{2^{\circ} \text { phase }}}+\frac{1}{R_{\mathrm{Gr}}^{2^{\circ} \text { hase }}}
$$

and:

$$
R=s /(\lambda \times A)
$$

Where $s$ is the thickness, $\lambda$ is the thermal conductivity and $A$ the heat flux passage area of the sample. In conclusion, a greater or lower amount of graphene during the second phase is inversely proportional to the thermal conductivity of the sample for a heat flux perpendicular to the coating (Figure 3), and so, to its diffusivity (Figure 4).

\section{Experimental results}

\subsection{GNP planes disposition}

On each sample the same amount of copper has been deposited, using for the 1st and 3rd electrodeposition the same

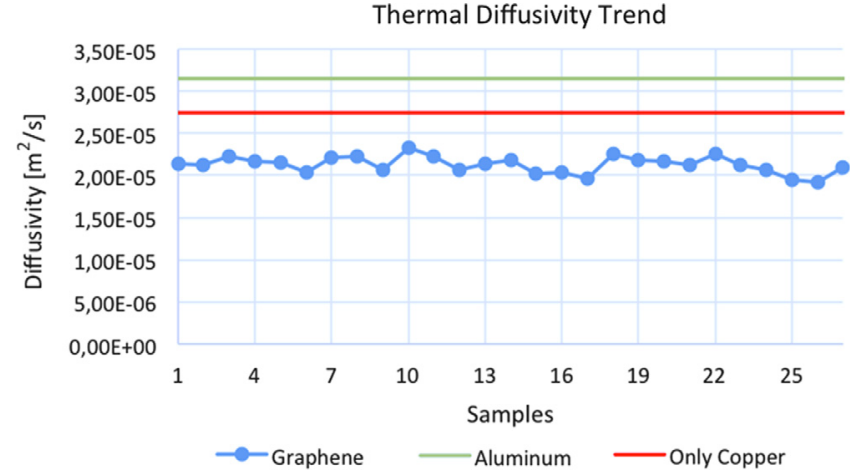

Figure 4. Experimental thermal diffusivity trend.

electrolytic current (2.3 A) and the same duration of process (40 min); in such a way, we could be sure that the diffusivity differences of each samples would have depended solely on the type of GNP deposited. Even for the 2nd electroplating phase, the duration of the electrodeposition was settled according to the currents imposed, in order to deposit every time the same amount of copper $(0.5 \mathrm{wt} \%)$, leaving the type of graphene as the only process variable. From the electrothermal model (Eqs. (1)-(3)) appears clear how an increase of the graphene amount would result in a greater reduction of the sample's thermal diffusivity (Figure 4).

Through the electro-thermal model was also possible to establish how most of the planes of GNP were arranged with respect to the substrate surface. For each iteration, the condition for which the thicknesses of the second phase remained congruent to those of the previous and following stages $(\sim 13 \mu \mathrm{m})$ corresponded to have thermal conductivity values of the GNP of about $4.85 \mathrm{~W} / \mathrm{mK}$, therefore very similar to those experienced by nano-graphites respect to a heat flow normal to the planes [42]. On the other hand, possible applications of the GNP conductivity similar to those shown along its plane $(\sim 1500-2000 \mathrm{~W} / \mathrm{mK})$ did not allow arriving at convergence. Experimentally observed disposition of GNP after the 2nd phase is in qualitative agreement with the disposition model of the GNP planes, as shown in Figure 5. This result inevitably implies that the arrangement of graphene platelets takes place parallel to the substrate on which they are deposited, manifesting an insulation behavior towards the heat flow generated by the flash method.

This arrangement of the GNP planes results congruent with the minimum energy principle, through which the planes disposition will happen according to the equilibrium more stable condition at the interface. In fact, the GNP are platelets characterized by wide single layers planar development compared to the multilayer height - Height $(H)$ : Length $(L)$ ratio $<0.0002-$ this implies that the condition with the single layers parallel to the substrate is the one able to make a higher number of bonds, and so the more stable one. In the end, graphene thermal coupling to other materials can depend strongly on the surface roughness, presence or absence of suspended regions in graphene layers, and methods of graphene preparation. 


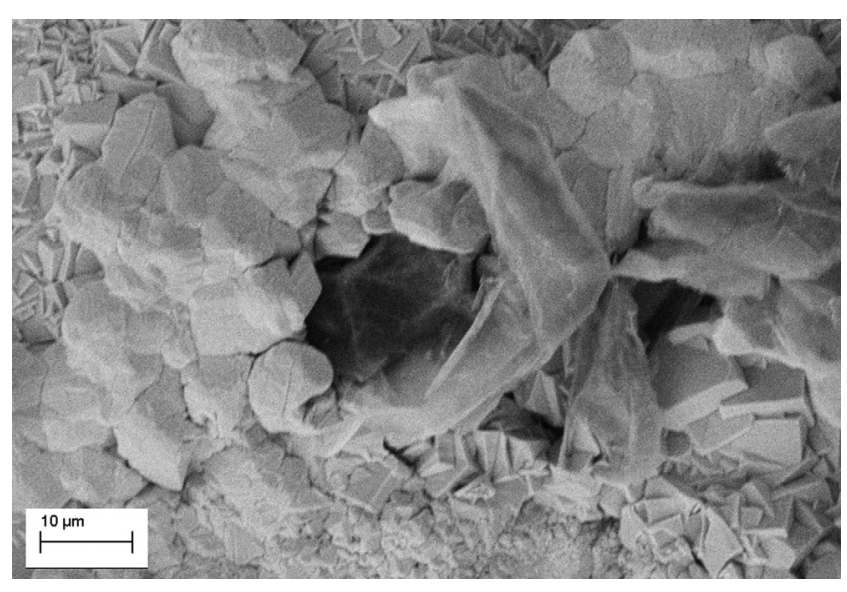

Figure 5. SEM image of the GNP arrangements during the 2nd phase.

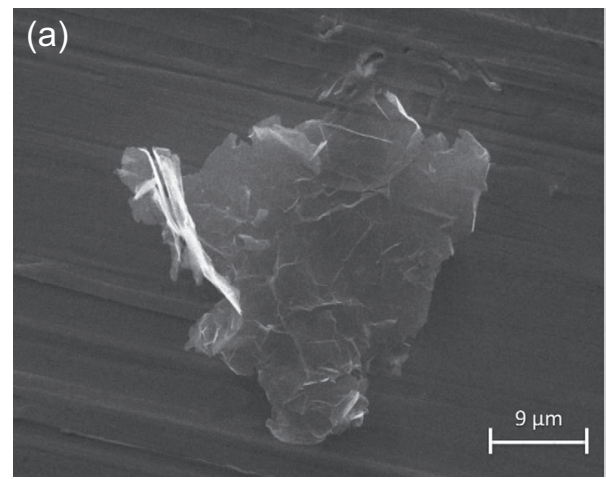

Figure 7. SEM image of: (a) G3N; (b) G2N.

\subsection{Mechanical properties}

As demonstrated by previous researches [2], there is a tight connection between the resistance of the foam and its density. Compression test results show that more the foam is dense and more it is resistant. Since the density of the produced samples is almost the same (around $513.5 \mathrm{~kg} / \mathrm{m}^{3}$, after the third phase), they show quite similar values for yield strength and deformation energy. As it can be seen from previous works $[2,3]$, the yield strength of third phase specimen enhances up to $232 \%$ in comparison to base aluminum foam. To demonstrate the effects of graphene electrodeposition on aluminum foam mechanical strength, two samples were chosen with very similar starting weight and density, such as $211 \mathrm{~kg} / \mathrm{m}^{3}$ and $2.4 \mathrm{~g}$. During electrodeposition, both samples were treated to obtain almost identical weight percentage change $(\Delta \mathrm{P} \%)$ in all phases. One of them was electrodeposited with copper only, while the other was electroplated with graphene and copper during its second phase. For the first and third phases a $\Delta \mathrm{P} \%$ of $100 \%$ was achieved, while for the second phase a $\Delta \mathrm{P} \%$ of $1.34 \%$ was tested. After compression tests, an improvement of around $25.7 \%$ was achieved by the sample treated with graphene. Figure 6 [3] shows the mechanical improvement.

The specimen electrodeposited only with copper is characterized by yield strength of $2.47 \mathrm{MPa}$ at a strain of 0.052 , while its densification strength is of $5.987 \mathrm{MPa}$ at a

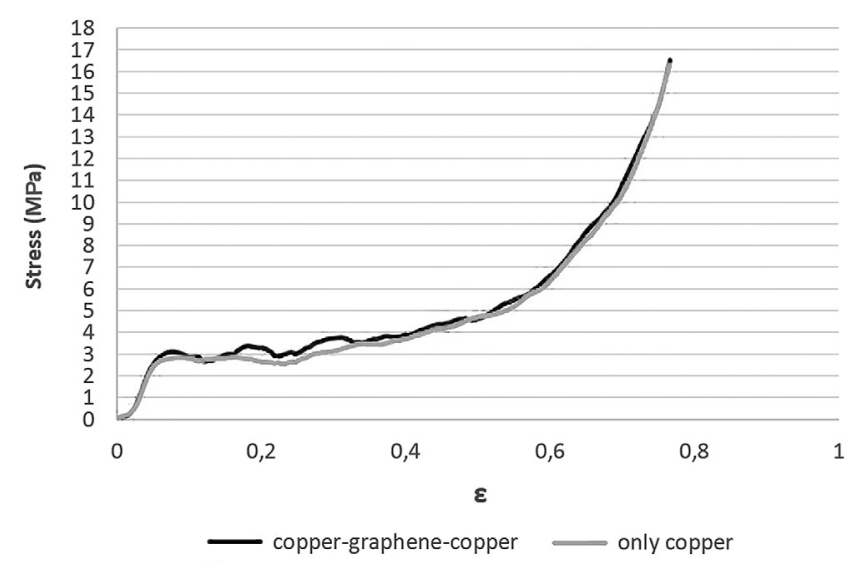

Figure 6. Stress strain curves comparison.

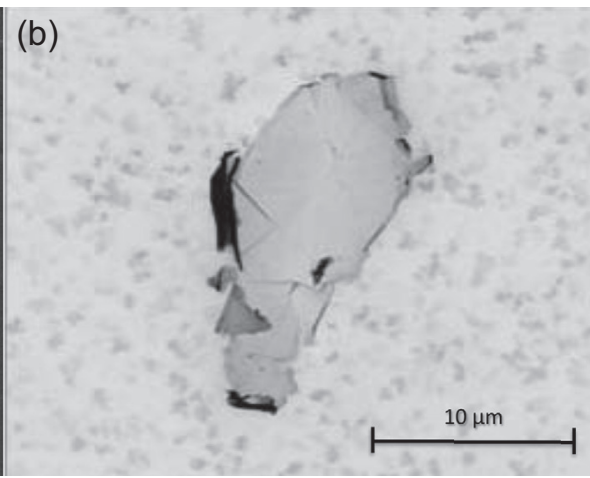

densification strain of 0.588 . The other sample, instead, achieved yield strength of $3.106 \mathrm{MPa}$ and densification strength of $5.803 \mathrm{MPa}$, respectively at the strains of 0.076 and 0.058 . The improvements achieved using graphene are related to the microstructure of its crystal lattice. In fact, the bindings between the atoms are very resistant, although the structure can be enough elastic to be deformed until $20 \%$.

\subsection{Heat conduction and nanoscale size effects on different GNP}

The main differences that have a direct influence on the thermal behavior of the two nano-particles studied $(\mathrm{G} 2 \mathrm{~N}$ and $\mathrm{G} 3 \mathrm{~N})$ regard their thickness $(H)$, their planar development $(L)$, their crystal lattice defects and their oxygen content. The presence of oxide on top of the graphene is known to cause defects in the graphene layer, but since both the nanoparticles studied present the same $\mathrm{C}: \mathrm{O}$ ratio $(30: 1)$, the differences of their thermal properties are mainly dependent by their geometrical dimensions and defects. With the increase of GNP thickness (number of atomic planes, $n$ ), two different cases need to be distinguished:

- thermal transport limited by intrinsic properties of the GNP lattice; 


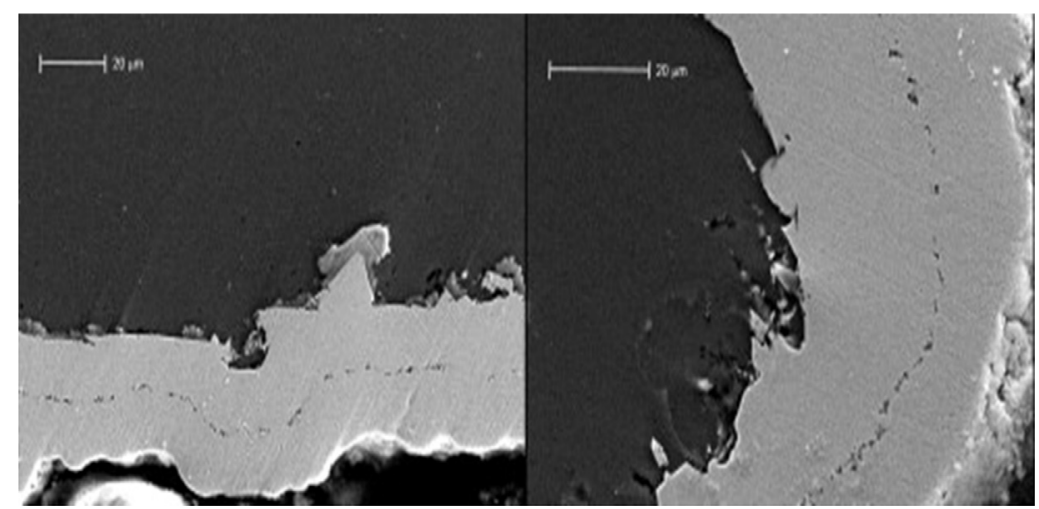

Figure 8. SEM image of the combined electrodeposition process of copper and graphene nano-platelets on aluminum foam substrate.
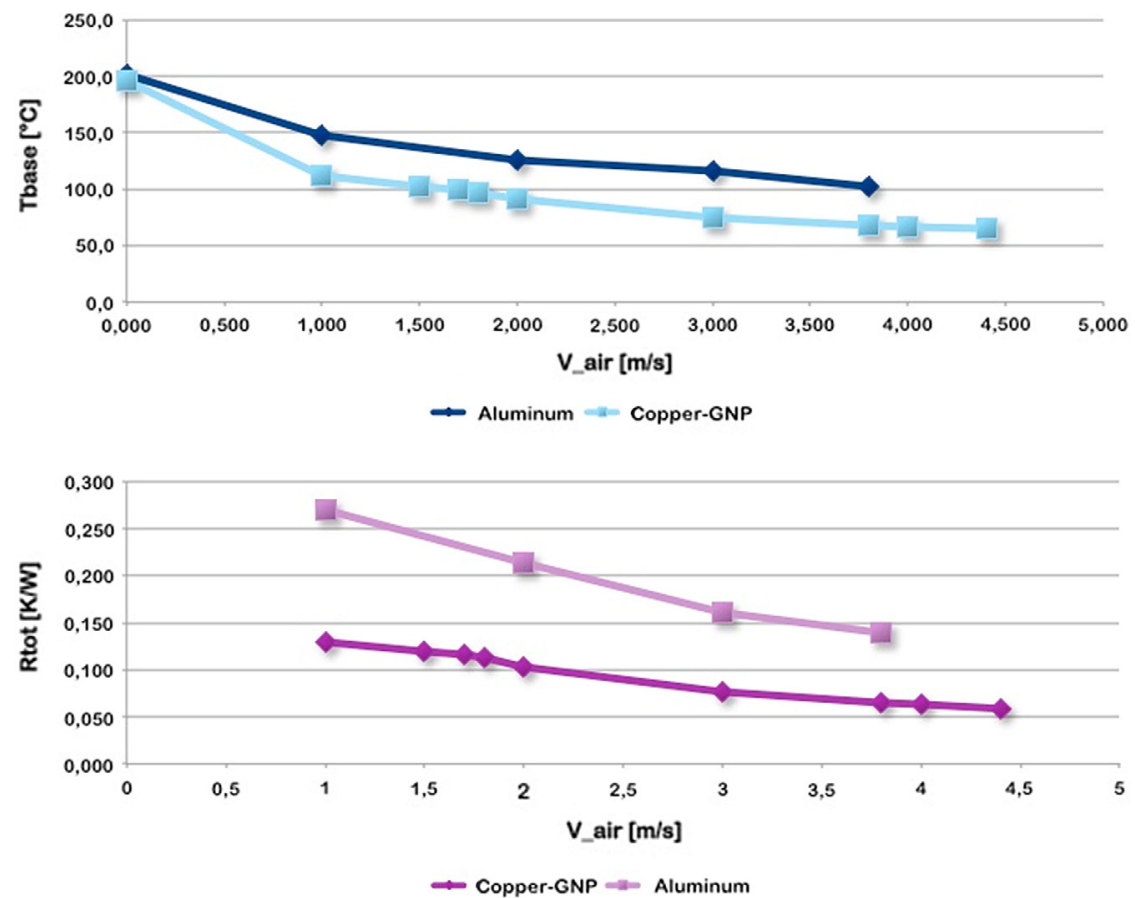

Figure 9. Steady state measurement in forced air convection of the foam with and without GNP.

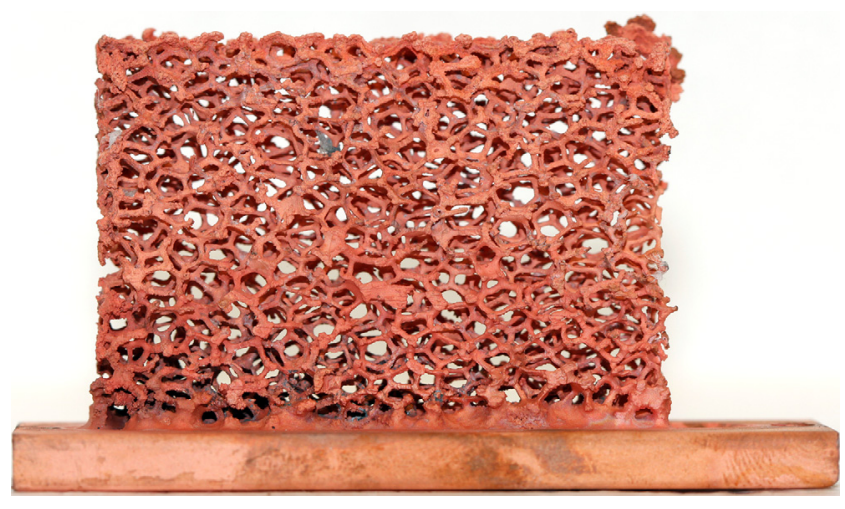

Figure 10. Image of the sample tested in un-steady state conditions.
- extrinsic effects, represented by phonon-boundary or defect scattering.

Through the optothermal Raman study [43] has been found that $\lambda$ of suspended uncapped FLG decreases with increasing $n$, approaching the bulk graphite limit. This evolution of $\lambda$ was explained by considering that if $n$ in GNP increases, the phonon dispersion changes and more phase-space states become available for phonon scattering leading to a decrease in $\lambda$. The two nanoparticles studied present defects in the crystal lattice due to the fabrication process. The presence of defects can alter the thermal behavior of the GNP because they improve the phonon scattering from the top and bottom boundaries. The inability to dispose of a lattice damage-free 


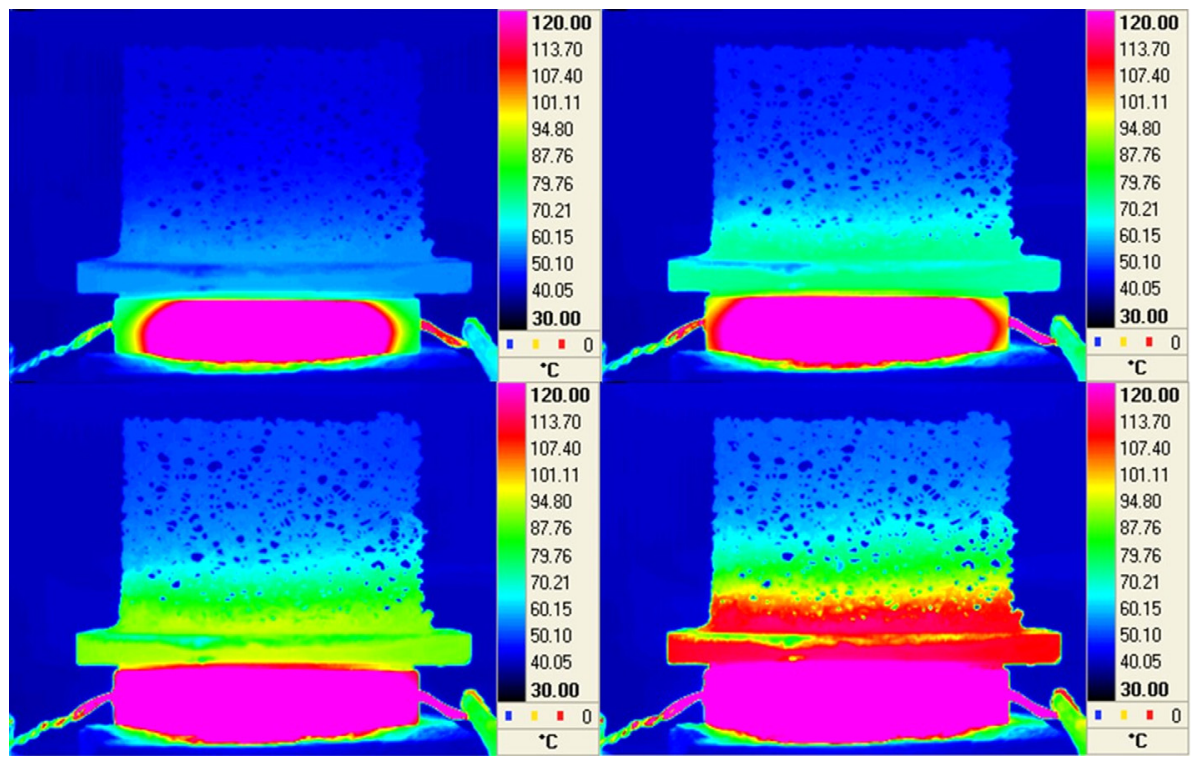

Figure 11. IR-thermal imaging analysis of the sample coated with G3N.

GNP, by which $n$ is constant through the whole area, generally lead to $\lambda$ values below the graphite limit (Figure 7).

To evaluate the thermal improvement due to the electrodeposition of GNP on 10 PPI aluminum foam (Figure 8 [3]), and therefore which one of the two different nanoparticles show a better thermal behavior, thermal analyses have been conducted.

To evaluate the improvement given by electrodeposition of GNP, several air-forced measures have been made. The use of forced air convection was due to the necessity of using the foams in their operating conditions as heat spreaders. An appropriate experimental apparatus has been designed in order to measure and control all the process variables. Direct steady state measurements on the samples were used for the comparison between the solution with and without graphene. The foam samples were all stacked between two copper fins with their base connected to a heat source. Forced air at the same inlet temperature with different speeds was passed through the samples, while the heat was transferred by conduction from the heat source to the base of the samples.

From the analysis of the results, the foam with electroplated GNP always shows a better thermal behavior compared to the one without GNP, for each inlet air velocity (Figure 9). This because the higher thermal conductivity of a graphene layer allows increasing the efficiency of the foam, overcoming the problems of thermal conductivity of the foam branches, let them reaching higher temperature in lesser time. For what concerns the thermal behavior of the two nanoparticles studied, IR un-steady thermal analyses were conducted without forced air convection. The prepared samples were brazed on a $70 \times 20 \times 5 \mathrm{~mm}$ copper base, connected to two $5 \mathrm{~W}$ each resistors (Figure 10).

The acquisition time has been set to $180 \mathrm{~s}$ for each sample and the main results are shown in Figure 9. The two GNP electrodeposited layers shows differences in terms of thermal diffusivity of the samples. From the IR-analysis

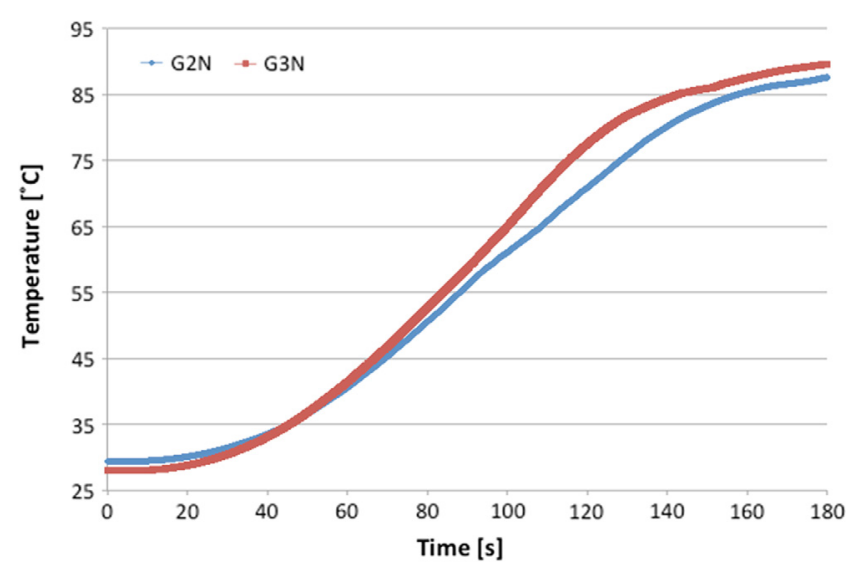

Figure 12. Thermal analysis of the electroplated foam with different GNP.

(Figure 11), it is clear how the layer with G3N presents a better thermal transient behavior with respects the one with G2N.

The temperatures reached by the G3N result higher than the ones reached by the $\mathrm{G} 2 \mathrm{~N}$ at the same time. This means that the thermal diffusivity (and so $\lambda$ ) of the G3N layer is superior to the one of the $\mathrm{G} 2 \mathrm{~N}$, as can be simply seen through the inclination of the curves in Figure 12. The thermal conductivity improvement obtained from the use of $\mathrm{G} 3 \mathrm{~N}$, compared to the $\mathrm{G} 2 \mathrm{~N}$, is found to be equal to $8.45 \%$.

This different thermal behavior of the G3N is mainly dependent by the lower presence of defects in the crystal lattice, as well as by a greater surface area, rather than in the number of layers. Recent non-equilibrium moleculardynamics calculations for graphene nanoribbons with $n$ from 1 to 8 [44] gave the thickness dependence $\lambda(n)$ in excellent agreement with the experiment [44], where $\lambda$ saturates near bulk graphite's value at $n \sim 7-8$. For that reason, 
since G2N and G3N present $n \sim 30$ and 20 respectively, the main difference in heat conduction strongly depends on the fabrication process and so by the defects produced in the lattice. Even the planar development $(L)$ plays an important role in heat conduction since $\lambda \cong \ln (L)$. In the end, the phonon-boundary scattering and disorder, resulting in characteristic $\lambda$ scaling with $H$, dominate heat transport in GNP. Nevertheless the scaling cannot follow exactly the same dependence as in crystalline films, because of the influence of disorder and changes of the material properties with $H$.

\section{Conclusions}

The flash method has clearly allowed the not destructive evaluation of the GNP presence in the thin layer electroplated. Through the electro-thermal analogy was possible to determine the disposition of the GNP planes during the combined electrodeposition process, subsequently validated through SEM analysis. Compression tests showed that the resistance and energy deformation of the specimens is proportional to their densities. In particular, graphene coating enhance the yield strength of the foam of almost $25.7 \%$. Steady state thermal analyses, through forced air convection, have permitted to evaluate the effective improvement of the GNP coating compared to the solution without GNP. The IR-thermo graphic analyses have allowed evaluating the different thermal conductivity behavior of the two nanoparticles, in unsteady state, finding in $\mathrm{G} 3 \mathrm{~N}$ the nanoparticles that maximize the efficiency of the foam. In the end, GNP coating permits to significantly improve the thermal conduction of the aluminum substrate, with low production costs, without burdensome methods and complex instrumentation, representing a new viable method to enhance the heat spreading.

Acknowledgements. The author thanks Mr. Massimiliano Della Millia and Mr. Massimiliano Tonti of the Department of Engineering of the University of Studies of Rome "Tor Vergata", Rome, Italy, for their continuous support throughout the research work.

The authors would like to acknowledge "NANESA s.r.l." of Arezzo for providing graphene nano-platelets.

\section{References}

1. R.L. McCreery, Advanced carbon electrode materials for molecular electrochemistry, Chem. Rev. 108 (2008) 2646.

2. A. Antenucci, S. Guarino, V. Tagliaferri, N. Ucciardello, Improvement of the mechanical and thermal characteristics of open cell aluminum foams by the electrodeposition of $\mathrm{Cu}$, Mater. Des. 59 (2013) 124-129.

3. A. Antenucci, S. Guarino, V. Tagliaferri, N. Ucciardello, Electro-deposition of graphene on aluminium open cell metal foams, Mater. Des. 71 (2015) 78-84.

4. C.E. Banks, T.J. Davies, G.G. Wildgoose, R.G. Compton, Electrocatalysis at graphite and carbon nanotube modified electrodes: edge-plane sites and tube ends are the reactive sites, Chem. Commun. 7 (2005) 829-841.
5. D. Chen, Q. Wang, J. Jin, P. Wu, H. Wang, S. Yu, H. Zhang, C.X. Cai, Low-potential detection of endogenous and physiological uric acid at uricase-thionine-single-walled carbon nanotube modified electrodes, Anal. Chem. 52 (2010) 2448.

6. L.N. Wu, X.J. Zhang, H.X. Ju, Detection of NADH and ethanol based on catalytic activity of soluble carbon nanofiber with low overpotential, Anal. Chem. 79 (2007) 453.

7. M. Zhou, J. Ding, L.P. Guo, Q.K. Shang, Electrochemical behavior of L-cysteine and its detection at ordered mesoporous carbon-modified glassy carbon electrode, Anal. Chem. 79 (2007) 5328.

8. M. Zhou, J.D. Guo, L.P. Guo, J. Bai, Electrochemical sensing platform based on the highly ordered mosoporous carbonfullerene system, Anal. Chem. 80 (2008) 4642.

9. H. Yaojuan, J. Juan, W. Ping, Z. Hui, C. Chenxin, Graphene - gold nanostructure composites fabricated by electrodeposition and their electrocatalytic activity toward the oxygen reduction and glucose oxidation, Electrochimica Acta 56 (2010) 491-500.

10. P.G. Klemens, Theory of the A-plane thermal conductivity of graphite, J. Wide Bandgap Mater. 7 (2000) 332-339.

11. H.O. Pierson, Handbook of carbon, graphite, diamonds and fullerenes: processing, properties and applications, Noyes Publications, Park Ridge, NJ, 2010.

12. C.Y. Ho, R.W. Powell, P.E. Liley, Thermal conductivity of the elements: a comprehensive review, J. Phys. Chem. Ref. Data 3 (1974) 1-30.

13. A.A. Balandin et al., Superior thermal conductivity of single layer graphene, Nano Lett. 8 (2008) 902-907.

14. S. Ghosh et al., Extremely high thermal conductivity in graphene: prospects for thermal management application in nanoelectronic circuits, Appl. Phys. Lett. 92 (2008) 151911.

15. I. Calizo, A.A. Balandin, W. Bao, F. Miao, C.N. Lau, Temperature dependence of the Raman spectra of graphene and graphene multilayers, Nano Lett. 7 (2007) 2645-2649.

16. S. Ghosh et al., Thermal properties of polycrystalline graphene films and reduced graphene-oxide films, MRS Proc. 2 (2010) 198.

17. K.S. Novoselov et al., Electric field effect in atomically thin carbon films, Science 306 (2004) 666-669.

18. K.P. Loh, Q. Bao, P.K. Ang, J.X. Yang, The chemistry of graphene, Mater. Chem. 20 (2010) 1.

19. D. Wei, Y. Liu, H. Zhang, L. Huang, B. Wu, J. Chen, G. Yu, Scalable synthesis of few-layer graphene ribbons with controlled morphologies by a template method and their applications in nanoelectromechanical switches, J. Am. Chem. Soc. 131 (2009) 11147.

20. M.D. Stoller, S. Park, Y. Zhu, J. An, R.S. Ruoff, Graphenebased ultracapacitors, Nano Lett. 8 (2008) 3498.

21. K. Kim, H.J. Park, B.C. Woo, K.J. Kim, G.T. Kim, W.S. Yun, Electric property evolution of structurally defected multilayer graphene, Nano Lett. 8 (2008) 3092.

22. C.G. Lee, X.D. Wei, J.W. Kysar, J. Hone, Measurement of the elastic properties and intrinsic strength of monolayer graphene, Science 321 (2008) 385.

23. C. Xu, X. Wang, J.W. Zhu, Graphene-metal particle nanocomposites, J. Phys. Chem. 112 (2008) 19841.

24. D. Wang, D. Choi, J. Li, Z. Yang, Z. Nie, R. Kou, D. Hu, C. Wang, L.V. Saraf, J. Zhang, I.A. Aksay, J. Liu, Self-assembled 
$\mathrm{TiO}_{2}$-graphene hybrid nanostructures for enhanced Li-ion insertion, ACS Nano 3 (2009) 907.

25. S.R.C. Vivekchand, C.S. Rout, K.S. Subrahmanyam, A. Govindaraj, C.N.R. Rao, Graphene-based electrochemical supercapacitors, J. Chem. Sci. 120 (2008) 9.

26. Y. Sui, J. Appenzeller, Screening and interlayer coupling in multilayer graphene field-effect transistors, Nano Lett. 9 (2009) 2973.

27. Y.J. Hu, J. Jin, H. Zhang, P. Wu, C.X. Cai, Graphene: synthesis, functionalization and applications in chemistry, Acta Phys.: Chim. Sin. 26 (2010) 2073.

28. S. Stankovich et al., Graphene-based composite materials, Nature 442 (2006) 282.

29. C. Mattevi, H. Kima, M. Chhowalla, A review of chemical vapor deposition of graphene on copper, J. Mater. Chem. 21 (2011) 3324-3334.

30. A. Reina, X. Jia, J. Ho, D. Nezich, H. Son, V. Bulovic, M.S. Dresselhaus, J. Kong, Large area few-layer graphene films on arbitrary substrates by chemical vapor deposition, Nano Lett. 9 (2009) 30-35.

31. O. Frank, M. Kalbac, Chemical vapor deposition (CVD) growth of graphene films, in: V. Skakalova, A. Kaiser (Eds.), Graphene, Woodhead Publishing, 2014, pp. 27-49.

32. L.A. Razak, D. Tobino, K. Ueno, Improvement of multilayer graphene quality by current stress during thermal CVD, Microelectron. Eng. 120 (2014) 200-204.

33. J.W. Suk, A. Kitt, C.W. Magnuson, Y. Hao, S. Ahmed, J. An, A.K. Swan, B.B. Goldberg, R.S. Ruoff, Transfer of CVD-grown monolayer graphene onto arbitrary substrates, ACS Nano 5 (2011) 6916-6924.

34. K. Boomsma, D. Poulikakos, F. Zwick, Metal foams as compact high performance heat exchangers, Mech. Mater. 35 (2003) 1161-1176.
35. W.J. Parker, R.J. Jenkins et al., A flash method of determining thermal diffusivity, heat capacity, and thermal conductivity, U.S. Navy Report USNRDL-TR-424, 1960.

36. P.G. Klemens, D.F. Pedraza, Thermal conductivity of graphite in basal plane, Carbon 32 (1994) 735-741.

37. P.G. Klemens, Theory of thermal conduction in the ceramic films, Int. J. Thermophys. 22 (2001) 265-275.

38. L.A. Jauregui et al., Thermal transport in graphene nanostructures: experiments and simulations, ECS Trans. 28 (2010) 73-83.

39. P.L. Kapitza, Collected Papers of P. L. Kapitza Vol. II, in: D. ter Haar (Ed.), Pergamon Press, Oxford, 1967, p. 581

40. Y.K. Koh, M.H. Bae, D.G. Cahill, E. Pop, Heat conduction across monolayer and few-layer graphenes, Nano Lett. 10 (2010) 4363-4368.

41. A.J. Schmidt, K.C. Collins, A.J. Minnich, G. Chen, Thermal conductance and phonon transmissivity of metal-graphite interfaces, J. Appl. Phys. 107 (2010) 1-5.

42. M.A. Raza, A. Westwood, A. Brown, N. Hondow, C. Stirling, Characterisation of graphite nanoplatelets and the physical properties of graphite nanoplatelet/silicone composites for thermal interface applications, Carbon 49 (2011) 4269-4279.

43. S. Ghosh et al., Dimensional crossover of thermal transport in few-layer graphene, Nature Mater. 9 (2010) 555-558.

44. W.R. Zhong, M.P. Zhang, B.Q. Ai, D.Q. Zheng, Chirality and thickness-dependent thermal conductivity of few-layer graphene: A molecular dynamics study, Appl. Phys. Lett. 98 (2011) 113107.

Cite this article as: Simoncini A, Ucciardello N \& Tagliaferri V: Thermal and mechanical improvement of aluminum open-cells foams through electrodeposition of copper and graphene. Manufacturing Rev. 2016, 3, 19. 